\title{
Article
}

\section{Les outils de pilotage des cultures : diversité de leurs usages et enseignements pour leur conception}

\author{
Marianne Cerf ${ }^{\mathrm{a}}$, Jean-Marc Meynard ${ }^{\mathrm{b}}$ \\ a Ergonome, INRA, UMR SADAPT, Bâtiment EGER, BP 1, 78850 Thiverval-Grignon, France \\ b Agronome, INRA, Département SAD, Bâtiment EGER, BP 1, 78850 Thiverval-Grignon, France
}

Faire le constat du décalage entre les conceptions à l'origine des outils et leurs utilisations pratiques est une chose assez partagée. Offrir une voie théorique et méthodologique pour intégrer la créativité des utilisateurs dans la conception de ces outils en est une autre, plus difficile. C'est ce que proposent les auteurs grâce à la coopération entre leurs points de vue disciplinaires, l'un et l'autre reconstituant une complexité qui va de la conception initiale d'un outil jusqu'à l'acte décisionnel final que représente son usage. On retrouve ici l'importance des démarches intégratives, prônées par la revue, à la fois sur le plan matériel et humain. L'enjeu de ces démarches est aussi opérationnel et illustre un cas d'école sur les relations entre la connaissance et l'action.

La Rédaction

\section{Mots-clés :}

interaction

conception-usage ; outils d'aide à la

décision;

conduite des cultures; interdisciplinarité
Résumé - La production d'outils d'aide à la décision est un domaine très actif, mais il est largement reconnu que de tels outils ne sont pas toujours utilisés comme leurs concepteurs l'ont prévu. Forts de ce constat, nous avons noué une collaboration entre une ergonome et un agronome, pour analyser la diversité des usages d'outils de conduite des cultures existants. Cette analyse met en évidence ce que nous proposons d'appeler des systèmes d'information et de conseil (SIC), organisations (temporelles et spatiales) de dispositifs humains et matériels qui permettent de construire et de diffuser les informations pour décider de la conduite locale de processus agroécologiques. Nous discutons comment cette notion peut être mise à profit pour faire évoluer la prise en compte des usages et des utilisateurs dans les processus de conception d'outils d'aide à la conduite de processus agroécologiques.

\section{Keywords:}

design-use interaction; decision support tools; crop monitoring; inter-disciplinarity
Abstract - Monitoring tools for crop management: deriving design guidelines from their diversity of uses. Although decision support tools are being produced in increasing numbers to monitor agronomic and ecological processes, most are however not used as anticipated by their designers. Based on this observation we initiated a joint study involving an ergonomist and an agronomist to analyse the manifold uses of existing tools designed to support crop monitoring. The analysis highlighted the existence of what we propose to call advisory and information systems (AIS). These are temporal and spatial networks of human and material arrangements which allow information to be developed and disseminated in order to make decisions about local monitoring of agro-ecological processes. We discuss the ways this concept can serve to better take into account uses and users during the design process of decision tools to support the monitoring of agro-ecological processes.

Auteur correspondant : M. Cerf, cerf@inapg.inra.fr 


\section{Introduction}

En agriculture, la production d'outils d'aide à la décision est un domaine $d^{\prime}$ innovation très actif ${ }^{1}$. Ainsi, se développent aujourd'hui de nombreux outils dédiés au raisonnement des apports d'intrants sur les cultures (engrais, pesticides...), autant pour répondre à la volonté des agriculteurs de réduire leurs coûts de production que pour intégrer les besoins, exprimés par divers acteurs de la société, de réduire les nuisances environnementales générées par l'agriculture intensive : modèles épidémiologiques et pièges à insectes pour prévoir les risques parasitaires et $\mathrm{n}^{\prime}$ appliquer des pesticides qu'en dernière nécessité ; modèles de culture et de dynamique de l'azote du sol ou indicateurs de nutrition des plantes pour un ajustement précis de la fertilisation azotée aux besoins des culture, etc. Ces outils, basés sur des connaissances agronomiques et biologiques de pointe, mettent souvent à profit l'évolution des biotechnologies (tests immunologiques) et des technologies de l'information (réflectomètres portatifs, techniques de simulation, images satellites...).

Du fait d'une mobilisation importante des acteurs de la recherche-développement (Inra, instituts et centres techniques agricoles, sociétés d'agrofourniture, coopératives, chambres d'agriculture, groupes de développement...), de nombreux outils ont ainsi vu le jour, et certains connaissent, ou ont connu, un réel succès. Mais plusieurs études passées (en particulier Jeuffroy et al., 1987; Cerf et Meynard, 1988; Felix et Reau, 1995) ont montré que leurs usages pouvaient différer notablement de ceux qu'avaient anticipés leurs concepteurs. La création d'usages non prévus par les concepteurs est bien connue des sociologues (par ex. Akrich, 1993), des ergonomes (par ex. Rabardel, 1995) ou des gestionnaires (par ex. Orlikowski et Robey, 1991) qui travaillent sur les processus d'innovation technique, c'est-à-dire sur les processus d'appropriation et d'adaptation de nouvelles techniques dans des organisations et des activités humaines. Nous ne revenons pas ici sur les raisons invoquées pour expliquer les différences entre usages prévus et usages réels. Notre propos est de mieux caractériser, en nous appuyant sur la confrontation des points de vue de l'agronome et de l'ergonome, la diversité des usages

\footnotetext{
${ }^{1}$ Ce travail repose sur une collaboration entre l'UMR d'Agronomie de Grignon et l'UMR SADAPT, réalisée tout d'abord à travers des mémoires d'étudiants (E. Emonet et $\mathrm{M}$. Taverne) dont les résultats ont donné lieu à des échanges avec les instituts techniques (ICTA). La collaboration s'est ensuite poursuivie avec le CETIOM (Centre technique interprofessionnel des oléagineux métropolitains) et $\mathrm{M}$. Taverne, recrutée à la suite de son mémoire, et a bénéficié du soutien d'actions transversales spécifiques de l'Inra. Cet article est le développement d'une conférence faite le 9 septembre 2004 lors des Entretiens du Pradel intitulés "Agronomes et innovations ».
}

observés, afin d'en tirer des enseignements pour intégrer cette diversité dès la conception des outils.

Dans la première partie de l'article, nous présentons succinctement l'approche que nous avons retenue pour ce travail ; dans la deuxième partie, nous décrivons la diversité des usages et des modes d'utilisation que nous mettons en évidence. Nous introduisons en troisième partie la notion de système d'information et de conseil (SIC) et mettons en évidence son intérêt pour l'analyse de la dynamique des outils. Nous proposons enfin, dans la quatrième partie, des pistes de travail conjoint entre agronome et ergonome pour la conception d'outils d'aide à la conduite des cultures ou, plus largement, d'aide au pilotage de processus agroécologiques.

\section{Les usages vus comme produits de l'activité constructive des acteurs}

Le mot «outil», objet fabriqué qui sert à faire un travail, fait simultanément référence ici :

- à un support matériel qui peut être, par exemple, un logiciel, une réglette, un analyseur, un kit de détection de maladie ou un piège à insectes ;

- aux procédures, formalisées dans des modes d'emploi, de recueil des informations ou des échantillons de plantes ou de sol qui alimentent le logiciel, le kit, etc. ;

- aux règles $\mathrm{d}$ 'interprétation du résultat de la mesure et d'agrégation des informations, qui participent très largement à la fiabilité de l'outil $^{2}$.

Comme le montre l'annexe, qui présente succinctement ceux que nous avons étudiés, les outils revêtent des formes très diverses. L'information pertinente est parfois recueillie par un comptage ou une observation réalisée au champ, parfois par des mesures faites en laboratoire sur des échantillons ramenés du champ. Les règles d'action qui utilisent cette information sont parfois totalement transparentes (exemple : seuil d'intervention), parfois intégrées dans des logiciels dont l'utilisateur ne connaît pas le contenu. Les logiciels sont parfois disponibles sur les ordinateurs des utilisateurs, qui peuvent se les approprier en réalisant des analyses de sensibilité ou en testant des situations familières, parfois consultables seulement sur minitel ou Internet, et, en général dans ce cas, opaques pour les utilisateurs.

Comme le souligne Akrich (1993), toute technique incorpore des représentations de l'utilisateur et de la situation d'usage, construites par le concepteur. Dans le cas

\footnotetext{
${ }^{2}$ Ces procédures et règles reflètent la manière dont le concepteur de l'outil se représente ses futurs usages. Mais, même si elles cadrent ceux-ci, nous ne les assimilons pas à des usages réels, c'est-à-dire mis en œuvre par les utilisateurs en situation d'action.
} 
des outils répertoriés en annexe, deux représentations sont clairement identifiables :

- celle d'agriculteurs prenant une décision instantanée à partir de données qu'ils recueillent et interprètent à l'échelle de la parcelle (la majorité des outils présentés en annexe) ;

- celle de conseillers élaborant un conseil à vocation régionale, pour diffuser ensuite des informations aux agriculteurs, en vue, là encore, d'une prise de décision instantanée par ces derniers (CLEAN, POSITIF).

Cependant, cette vision de la décision vue comme un processus instantané a été largement battue en brèche: de nombreux travaux, dans la lignée de ceux de Simon sur la rationalité procédurale, ont montré que la décision est un processus qui s'étale dans le temps, d'une part parce que toute l'information pertinente n'est pas disponible à tout moment, d'autre part parce que, pour limiter l'incertitude dans laquelle il se trouve, le décideur est souvent conduit à anticiper, à préparer un choix qu'il devra faire plus tard (voir, par exemple, Hoc, 1989; Sebillotte et Soler, 1988; Amalberti, 1999). Les écrits scientifiques produits par les concepteurs à propos des outils qu'ils ont élaborés laissent le plus souvent dans l'ombre cette question de l'organisation temporelle des informations issues des outils $^{3}$.

De la même façon, la dichotomie entre des outils pour une décision à la parcelle et des outils pour un conseil régional semble simpliste. Elle est, avant tout, liée à la manière dont les agronomes se représentent la variabilité des processus agroécologiques et, partant, des interventions techniques à réaliser pour les piloter. Ainsi, le développement des maladies aériennes des céréales dépend avant tout de facteurs climatiques, peu variables d'une parcelle à l'autre au sein d'une petite région. Un modèle climatique (CLEAN, POSITIF, $c f$. Annexe), nourri par des données météorologiques, permet ainsi de diffuser au niveau régional des conseils sur l'opportunité d'appliquer un traitement fongicide. Au contraire, la biodisponibilité de l'azote du sol est réputée très variable, même au sein d'une petite région et pour une année donnée (Meynard et al., 1997). Les outils prédictifs fonctionnent donc au niveau d'unités écologiquement homogènes (de taille généralement inférieure à la parcelle agricole), et s'en servir pour fixer une dose d'engrais au niveau d'une parcelle agricole, parfois en elle-même assez hétérogène, résulte déjà d'un compromis entre le faisable et un «idéal agronomique». La question de la concordance entre les échelles auxquelles les outils fournissent des

\footnotetext{
${ }^{3}$ Ce n'est pas toujours vrai. La méthode de fertilisation JUBIL prévoit, par exemple, une décision en deux temps : calcul en fin d'hiver d'une dose d'engrais azoté, apportée à la culture au début du printemps ; quelques semaines plus tard, plusieurs mesures de la teneur en nitrate des plantes, pour décider d'apporter ou non un complément de fertilisation (Justes, 1993).
}

informations et celles auxquelles les acteurs les utilisent mérite donc d'être posée.

Il y a lieu, enfin, de s'interroger sur le rapport entre la décision et l'action : in fine, c'est l'action qu'il s'agit d'ajuster à la situation. Or, l'action n'est pas réductible à la décision et les ressources disponibles pour agir ne sont pas qu'informationnelles. Les approches développées à partir de l'analyse de l'activité déployée pour agir en situation, qu'elles le soient en sociologie, en anthropologie ou en ergonomie, montrent aussi les limites de cette vision réduisant l'acteur à un système de recueil et de traitement de l'information. Ainsi, les théories de l'action et de la cognition située (Suchman, 1987; Hutchins, 1995) insistent sur la façon dont l'acteur s'adapte, en situation, aux ressources disponibles, et agit en s'appuyant sur ces ressources sans avoir nécessairement un plan d'action prédéfini. Les théories de l'activité mettent quant à elles en avant l'activité constructive de l'acteur, c'est-à-dire sa capacité à créer les ressources de son action (Samurçay et Rabardel, 1995), en attribuant des propriétés aux ressources disponibles, en lien avec ses intentions d'agir sur la situation. Au-delà, l'utilisation des outils est source de développement ${ }^{4}$ de l'activité et de celui qui la met en œuvre. Les outils sont ainsi pris comme des ressources à la fois pour agir et pour faire évoluer les modalités et le sens de l'action, conformément à la façon dont les acteurs voient leur travail et ses résultats attendus.

Dans cette perspective, la conception se poursuit dans l'usage (Béguin et Rabardel, 2000), celui-ci engendrant un apprentissage qui fait du travail du concepteur au mieux une hypothèse, qui sera validée, réfutée ou remise en mouvement. Notre travail s'inscrit bien dans la reconnaissance de cette créativité des utilisateurs, qui se traduit par des situations d'utilisation différentes de celles anticipées par les concepteurs. Il s'agit alors de caractériser ces situations et leur diversité, pour en tirer profit dans le travail de conception, mais aussi de s'interroger sur la possibilité de construire conjointement les outils et leurs usages en reconnaissant aux utilisateurs un statut dans le processus même de conception. En d'autres termes, il s'agit de favoriser, durant la conception, des apprentissages mutuels entre concepteurs et utilisateurs potentiels (Kyng, 1995 ; Bødker et Grønboek, 1996 ; Bratteteig, 1997 ; Béguin, 2003).

Les usages ont été analysés, à partir de plusieurs enquêtes de terrain, auprès d'agriculteurs et de prescripteurs (en Lorraine et Eure-et-Loir principalement) pour les outils présentés en annexe. Une étude de la presse agricole ${ }^{5}$ et un retour d'expérience issu de notre

\footnotetext{
${ }^{4}$ Cette notion renvoie à la transformation des buts, des façons de faire, des catégories d'analyse du réel, etc. Elle repose sur des apprentissages qui s'opèrent ici à travers l'utilisation des outils.

${ }^{5}$ Collection des revues Cultivar, Oléoscope, Perspectives agricoles, Horizons 28, sur la période 1994-2004.
} 
Encadré. L'organisation de la complémentarité entre outils pour la fertilisation azotée en Eure-et-Loir (2001).

En janvier, la chambre d'agriculture organise une campagne de mesure de l'azote minéral du sol dans les parcelles de blé des agriculteurs volontaires. Les prélèvements de sol sont faits par les agriculteurs, envoyés à un laboratoire d'analyses, et les résultats sont retournés aux agriculteurs avec un calcul de dose d'engrais réalisé par le logiciel AZOBIL.

L'ensemble des mesures d'azote du sol est synthétisé par les techniciens, et les valeurs moyennes par précédent et type de sol sont diffusées en février par les conseillers de la chambre d'agriculture à l'ensemble des agriculteurs. L'information à la parcelle devient ainsi une information régionale, qui permet de faire le calcul de dose d'engrais sur les parcelles où les agriculteurs n'ont pas fait de mesure.

Les techniciens diffusent aussi un tableau de doses d'engrais recommandées, établi à l'aide d'AZOBIL sur la base des références régionales, une dose type étant proposée, pour chaque région agricole, par type de sol et précédent cultural. Les agriculteurs peuvent ainsi bénéficier, pour fixer leurs doses d'engrais, des mesures d'azote minéral et de l'appui de l'outil AZOBIL de trois manières différentes : calcul parcellaire sur la base de mesures parcellaires; calcul parcellaire sur la base des moyennes régionales d'azote minéral; grille régionale.

Ces doses totales, proposées en février ou mars, peuvent être ajustées en avril-mai par la mesure de la teneur en nitrate du jus de base de tige (JUBIL) ou de la teneur en chlorophylle des feuilles (méthode Hydro N-Tester), les deux outils étant diffusés dans la région (chambre d'agriculture et groupes coopératifs). L'agriculteur qui veut utiliser l'un de ces outils diminue de $40 \mathrm{~kg}$ N/ha la dose recommandée à l'étape précédente, et réalise pendant la montaison un ou des diagnostics de nutrition azotée de la culture. Les techniciens se tiennent informés des résultats de ces suivis (ils constituent des intermédiaires obligatoires pour l'utilisation du H N-T), et s'en servent pour adapter le conseil qu'ils donnent aux agriculteurs qui n'utilisent pas ces outils («Cette année, les blés valoriseront bien un complément d'azote»).

Les messages des prescripteurs sont non seulement transmis oralement lors d'entretiens individuels ou de réunions d'information, mais sont également diffusés dans les journaux professionnels ou dans des notes d'information; chaque prescripteur sait donc ce que diffusent les prescripteurs d'autres structures.

participation à la conception de plusieurs outils de raisonnement (la fertilisation azotée pour l'un d'entre nous, des traitements contre le sclerotinia pour l'un et l'autre) ont complété le dispositif d'enquête.

\section{Utilisateurs, usages et utilisation des outils}

\section{Les utilisateurs et les usages spécifiques qu'ils créent}

D'après Fixari et Spira (1998), la méthode JUBIL, conçue pour des utilisateurs agriculteurs, a surtout été utilisée par des conseillers agricoles. Nos résultats montrent que de nombreux outils sont dans le même cas. Ainsi, les prescripteurs sont les principaux utilisateurs des logiciels de calcul des doses d'engrais : par exemple, le calcul de la dose d'azote avec le logiciel AZOBIL est un service offert par les laboratoires d'analyses de terre aux agriculteurs qui commandent une analyse d'azote minéral en sortie d'hiver. Plus généralement, les outils basés sur la méthode du bilan prévisionnel, initialement destinés aux agriculteurs (ITCF, 1978), restent peu utilisés par ceux-ci. Ce sont les prescripteurs qui s'en servent le plus, en particulier pour élaborer chaque année des conseils de fertilisation sur des cas types (Encadré), diffusés dans la presse agricole. Un tel glissement de ces outils vers les conseillers est cohérent avec la manière dont les agriculteurs raisonnent leurs doses d'engrais azoté : Jeuffroy et al. (1987), Cerf et Meynard (1988), Felix et Reau (1995) ont montré que la plupart des agriculteurs se fixent des « doses de référence », valables en année moyenne sur des situations bien maîtrisées (par exemple, la combinaison "précédent $\times$ type de sol » la plus représentée dans leur exploitation). Leur demande de conseil porte sur les règles d'ajustement de cette dose, en plus ou en moins, en fonction des années et des situations agronomiques.

La cuvette jaune, outil de base du raisonnement de la protection contre les ravageurs du colza, est également $l^{\prime}$ objet de créations d'usages. Le concepteur ${ }^{6}$ conseille de placer une cuvette sur chaque parcelle et donne des indications précises sur le positionnement du piège au sein de la parcelle (Hébinger, 1994). La présence d'insectes dans le piège à une période de sensibilité des plantes déclenche un comptage du nombre d'insectes sur les plantes et, si un seuil défini est dépassé, un traitement. Dans la réalité, ainsi que le montre une enquête réalisée en Lorraine auprès de 45 agriculteurs et 18 prescripteurs, la plupart des agriculteurs utilisateurs de la cuvette jaune n'en positionnent qu'une et raisonnent le traitement de toutes leurs parcelles de colza à partir des informations recueillies grâce à cette cuvette (Emonet, 1998). Alors que seuls certains agriculteurs utilisent la cuvette jaune, tous les prescripteurs (conseillers de chambre d'agriculture ou de coopératives) se constituent des "réseaux de cuvettes », dont la surveillance leur permet d'annoncer la présence d'un ravageur dans leur secteur et d'élaborer leur conseil de traitement. Ces réseaux composites associent des cuvettes installées par le prescripteur dans des endroits qu'il a choisis et des cuvettes installées par des agriculteurs sur leurs parcelles, dont le conseiller recueille les résultats. Cette utilisation de l'outil en réseau,

${ }^{6}$ Le CETIOM, centre technique agricole. 
au niveau régional, s'accompagne de fait de nouvelles règles $\mathrm{d}$ 'action.

Ainsi, en changeant d'utilisateur, les outils changent également d'usage et d'échelle d'utilisation. On peut légitimement s'interroger sur leur fiabilité dans ces nouveaux usages, alors qu'ils ont été conçus et validés pour d'autres usages, d'autres échelles et d'autres règles d'action.

\section{Une diversité d'usages pour une même catégorie d'utilisateurs}

Au-delà de cette diversité des catégories d'utilisateurs (identifiées par des métiers différents), il existe également, pour une catégorie d'utilisateur donnée, voire pour un individu donné, une grande diversité dans les usages d'un même outil. Ainsi, Emonet (1998) fait état de situations où des agriculteurs utilisent les informations issues de l'analyse de teneur en nitrate des tiges de blé, ou de l'observation du contenu des cuvettes jaunes, non seulement pour alimenter leurs décisions d'intervention (usage prévu et normé par les concepteurs), mais également pour vérifier a posteriori la pertinence de leurs décisions.

Cette diversité d'usages d'un même outil s'observe aussi chez les prescripteurs. L'enquête d'Emonet (ibid.) montre que, quelques années après sa diffusion, ces derniers utilisent JUBIL de quatre manières différentes. Tout d'abord (i) pour réaliser un conseil direct aux agriculteurs, au niveau de la parcelle, sur des situations atypiques (retournement de prairie, fort apport de fumure organique, ou bien l'année qui suit une sécheresse ayant occasionné de forts excédents d'engrais). Cet usage est explicitement prévu par les concepteurs de JUBIL ${ }^{7}$ (Justes, 1993). Ensuite (ii) pour faire une animation technique, les conseillers organisant des « rendez-vous JUBIL », réunions de groupes d'agriculteurs sur le terrain, pour présenter la méthode et rappeler la nécessité de différencier la fertilisation selon les parcelles et/ou de fractionner l'engrais. Puis (iii) pour réaliser un ajustement interannuel du conseil en comparant des résultats obtenus par l'outil sur un réseau une année donnée à ceux obtenus les années précédentes sur des réseaux équivalents. Enfin, (iv) comme référence pour l'évaluation de la fiabilité d'autres outils, plus récents, susceptibles d'être intéressants. Au moment du développement du Hydro N-Tester, qui visait le même créneau d'utilisation que JUBIL tout en étant plus simple d'emploi, de nombreux prescripteurs ont mis en place des expérimentations pour comparer les résultats des deux outils. Une telle évaluation des pratiques et des innovations, de la

\footnotetext{
${ }^{7}$ JUBIL a été conçu comme un outil adapté aux situations où la forte fourniture du sol ne permettait pas de prévoir précisément la dose d'engrais à l'aide de la seule méthode du bilan prévisionnel.
}

part des agriculteurs comme des conseillers, fait donc des outils une réelle source d'apprentissage.

\section{La complémentarité entre les outils}

Parmi les usages créés par les utilisateurs, une place particulière doit être réservée à ceux qui reposent sur l'organisation d'une complémentarité entre des outils conçus initialement pour être utilisés seuls.

Trois types de complémentarité peuvent être identifiés :

- spatiale : le premier outil apporte des informations au niveau régional, qui permettent d'alléger le coût d'utilisation d'un deuxième outil, mis en œuvre à l'échelle de la parcelle. Les modèles épidémiologiques régionaux (par exemple POSITIF ou CLEAN, $c f$. Annexe) qui simulent l'évolution du risque de maladies sont souvent utilisés ainsi, comme l'a mis en évidence Taverne (2000). Le modèle permet de déclencher la surveillance des parcelles et indique les zones de la région où celle-ci est la plus nécessaire ;

- temporelle : le premier outil fournit une information précoce qui permet soit d'anticiper l'intervention et de réaliser les achats d'intrants en morte saison, soit de préparer l'utilisation d'un deuxième outil. C'est ainsi, par exemple, qu'est utilisée la grille de risque " piétin-verse du blé », diffusée par ARVALIS-Institut du végétal, qui sert à identifier les parcelles à surveiller;

- d'apprentissage : un outil prépare l'action, un autre (ou le même, détourné) permet d'en juger les résultats; le deuxième outil valide le premier ou permet de l'améliorer. Par exemple, il n'est pas rare qu'un agriculteur, s'appuyant sur un outil pour décider d'un traitement phytosanitaire, laisse une bande-témoin non traitée. Dans les semaines qui suivent, il observe l'évolution des symptômes parasitaires sur la bandetémoin. Lors de la moisson, grâce à des capteurs de rendement, il mesure le rendement sur le témoin et sur une bande contiguë traitée, évaluant ainsi son intervention et la pertinence des outils qu'il a mobilisés.

En fait, au niveau d'une région, s'organisent autour des outils une production et un échange d'informations, qui mobilisent agriculteurs et conseillers et alimentent un processus de décision distributif (Encadré). L'information issue des outils circule donc dans un réseau associant agriculteurs et techniciens, chacun recevant de l'information de plusieurs autres, l'utilisant pour prendre des décisions soit d'intervention, soit de prescription (Taverne, 2000). La prise en compte de ces réseaux, qui associent des dispositifs matériels, des compétences et des coordinations entre acteurs, nous paraît essentielle pour définir les conditions d'utilisation et de conception des outils. 
Pour en rendre compte, nous proposons le concept de «système d'information et de conseil ».

\section{La notion de système d'information et de conseil}

\section{Définition}

Nous appelons «système d'information et de conseil» (SIC) une organisation (temporelle et spatiale) des dispositifs humains et matériels qui permettent de construire et de diffuser les informations pour décider de la conduite locale de processus agroécologiques.

Une telle notion reconnaît, comme cela a été mis en avant au niveau de l'agriculteur avec la notion de modèle d'action (Sebillotte et Soler, 1988), que toute action de transformation des processus agroécologiques est précédée par une étape de préparation de $1^{\prime}$ action ${ }^{8}$. Cette étape, que nous qualifions d'activité décisionnelle ${ }^{9}$, comporte à la fois des dimensions matérielles (achat d'intrants, préparation et localisation de matériel ou d'animaux, prélèvements d'échantillons pour les analyses, mobilisation d'un ordinateur pour stocker et interpréter des données, etc.) et des dimensions immatérielles liées aux processus cognitifs en jeu (intelligence de la situation, conception de solutions et choix d'une solution satisfaisante, évaluation). Cependant, contrairement au modèle d'action qui est propre à chaque agriculteur, cette activité décisionnelle est distribuée, c'est-à-dire qu'elle est réalisée par plusieurs acteurs et dispositifs matériels. Dès lors, la circulation des informations entre les acteurs devient un élément important à considérer, de même que les opérations de traduction qui s'opèrent lors de cette circulation des informations. De plus, un SIC ne se réduit pas à un système de recueil et de traitement des informations. Les formes concrètes qu'il prend sont dépendantes des enjeux dont sont porteurs les acteurs participant au fonctionnement du système et de leurs représentations de l'activité de contrôle des processus agroécologiques. Enfin, la notion de SIC doit être resituée dans un espace de décision et dans une temporalité des activités décisionnelles. Les acteurs qui participent à ce système n'ont pas les mêmes espaces de «concernement» ni les mêmes exigences et contraintes temporelles. L'espace de

\footnotetext{
${ }^{8}$ Ce présupposé n'est pas lié à des hypothèses sur le traitement de l'information par l'acteur, qu'il soit individuel ou collectif, mais aux caractéristiques de sa situation d'action : la relative lenteur de l'évolution des processus, mais aussi la nécessité d'agir à certains moments-clés pour éviter une évolution que l'acteur jugerait défavorable, créent les conditions de l'apparition de cette étape.

${ }^{9}$ Nous préférons parler d'activité décisionnelle plutôt que de processus de décision pour insister sur le fait que des dispositifs humains et/ou matériels sont mobilisés pour recueillir et traiter les informations.
}

l'agriculteur est celui des surfaces qu'il exploite, celui des conseillers est moins clairement délimité (bassin d'approvisionnement pour les technico-commerciaux des coopératives, petite région pour le conseiller de chambre d'agriculture, région administrative pour l'ingénieur du Service régional de la protection des végétaux... ). Le rapport au temps sépare tout autant l'agriculteur, qui s'organise en fonction des dates d'achat d'intrants et d'interventions au champ (Soulard, 2005), et le conseiller, tenu par les sollicitations des agriculteurs et les dates de disponibilité des informations qu'il collecte et synthétise.

\section{Les nouveaux outils, source d'évolution des SIC}

L'adoption d'un nouvel outil par l'un des acteurs du réseau modifie la nature des informations disponibles et transforme les flux d'informations, les coordinations entre acteurs, voire les compétences nécessaires pour l'activité décisionnelle et pour l'action. Ainsi, les outils de pilotage de la fertilisation (JUBIL, Hydro N-Tester...) ont changé à la fois le calendrier des interventions et celui de la production d'informations. De même, l'accroissement des connaissances sur l'épidémiologie des maladies a permis au Service de la protection des végétaux de modifier le fonctionnement de ses réseaux d'avertissements agricoles, les modèles donnant une indication du risque plus précoce et plus aisément extrapolable que les réseaux d'observateurs.

Plus radicalement, les coordinations évoluent en lien avec le développement d'un secteur marchand autour des informations pour le pilotage des cultures. Les outils de pilotage de la fertilisation azotée et les kits de détection de maladies ont ainsi ouvert en grande culture la voie au conseil personnalisé payant, qui se développe aujourd'hui très rapidement. Les financements publics ou mutualisés de la recherche-développement agricole ne permettent plus de couvrir les coûts de l'innovation et de la maintenance des outils les plus sophistiqués, alimentés par des informations très onéreuses à recueillir et à traiter (voir par exemple Farmstar, proposé par ARVALIS - Institut du végétal, qui utilise des informations acquises par un satellite). L'utilisation de l'outil étant facturée, le réseau devient, beaucoup plus formellement, celui d'une relation client-fournisseur et l'interconnexion entre les réseaux des différents fournisseurs, si elle continue à exister, est beaucoup moins visible. Pour faciliter leur maintenance, les outils les plus complexes ne sont pas diffusés, mais seulement consultables par Internet : 1'emprise géographique du réseau en est décuplée; les informations saisies par les utilisateurs sont stockées dans une base de données qui permet d'enrichir le référentiel de l'outil : les clients fournissent ainsi gratuitement au prestataire de service des informations agronomiques qui constituent de fait pour celui-ci une richesse qu'il peut s'approprier 
en la stockant. On retrouve toutes les caractéristiques du SIC, mais sa forme a profondément changé.

\section{Les SIC, un point d'appui pour la conception de nouveaux outils}

Les SIC peuvent également devenir des lieux privilégiés pour le développement de nouveaux outils. Ainsi, en Aunis et en Lorraine, on a assisté dans les années 1990 à l'émergence d'une nouvelle écriture du calcul des doses d'engrais azoté (Meynard et al., 1997, logiciel PCAzote. . .), basée sur une modélisation de la variabilité de l'azote absorbé par les cultures non fertilisées (Plas, 1992 ; Limaux et al., 1993) et du coefficient apparent d'utilisation de l'engrais (Limaux et al., 2001). L'avantage de ce formalisme est que les principaux paramètres du calcul sont mesurables aisément par les prescripteurs sur leurs expérimentations, ce qui leur procure une grande autonomie pour adapter leur outil et prendre en compte les spécificités locales. Il semble pertinent de mettre l'émergence de cette innovation en relation avec les caractéristiques des SIC locaux de cette époque : une forte mobilisation collective des conseillers autour de la fertilisation azotée, se traduisant en particulier par une intense activité d'expérimentation; $1^{\prime}$ absence ${ }^{10}$ de dispositif régional de mesure des quantités d'azote dans le sol en fin d'hiver, indispensable à la mise en œuvre de l'écriture classique de calcul des fumures azotées par le bilan prévisionnel de Rémy et Hébert (1977) et le logiciel AZOBIL.

\section{Les SIC et l'accompagnement de l'outil}

Les SIC peuvent être aussi bien un frein qu'un support aux apprentissages que développent les agriculteurs et les conseillers lors de l'usage de nouveaux outils. Dans les exemples touchant à l'organisation du conseil de fertilisation azotée autour de JUBIL, en Lorraine ( $c f$. paragraphe précédent) ou en Eure-et-Loir (Encadré), le travail en groupe autour de l'outil a permis d'accompagner l'apprentissage de son mode d'emploi et de ses propriétés, tout en accroissant les compétences des agriculteurs sur le fractionnement de l'engrais (voir aussi Fixari et Spira, 1998). À l'inverse, les apprentissages peuvent s'avérer limités dans le cas d'un système opaque, mis en place par un prestataire souhaitant développer un service payant. Le prestataire reste propriétaire de l'interprétation qui justifie la facturation du service : une pérennisation de l'asymétrie des connaissances est ici recherchée, puisqu'elle est à l'origine même de la création du marché.

\footnotetext{
${ }^{10}$ Due à une forte fréquence de sols très caillouteux où il est difficile d'enfoncer une tarière pour mesurer l'azote contenu dans les couches les plus profondes.
}

Par ailleurs, le SIC peut permettre d'assurer la maintenance de l'outil et son adaptation à des gammes de situations dans lesquelles il n'a pas été validé. Par exemple, la méthode JUBIL a été mise au point sur la variété Thésée (Justes, 1993) et sa robustesse n'a pas été testée dans un premier temps sur d'autre variétés, alors que l'indicateur s'est avéré à l'usage sensible à ce facteur. Il a donc fallu mettre en place, chaque année, des expérimentations pour savoir à quelle abaque d'interprétation rattacher chaque nouvelle variété (Laurent et al., 1996). Cette dépendance de l'outil à l'égard du SIC peut poser problème, car la maintenance de l'outil est coûteuse, et, dès qu'elle ne sera plus assurée, l'outil sera condamné. Comme le soulignent Meynard et al. (2002), il serait souhaitable de conduire, dès la conception de l'outil, une réflexion sur la façon dont sa maintenance sera prise en compte dans les SIC. Ainsi, dans certains travaux récents, la facilité d'adaptation de l'outil à de nouvelles variétés a été placée en tête du «cahier des charges » lors de la conception : c'est le cas, par exemple, de l'outil BETHA (Loyce et al., 2001), qui vise à aider au choix de l'itinéraire technique du blé.

\section{Discussion : conséquences pour la conception des outils}

L'ensemble des résultats présentés démontre qu'il existe bien un décalage important entre les usages des outils et les représentations qu'en ont leurs concepteurs. Cette diversité d'usages témoigne d'une créativité qui n'est pas sans risque (usages non validés, extrapolations abusives). Mais il est clair que l'on gagnerait à mieux mobiliser cette créativité dans la conception des outils. Nous proposons de le faire en nous appuyant sur la notion de SIC : (i) la grande majorité des outils d'aide à la conduite des cultures s'appuie aujourd'hui sur une représentation de l'activité décisionnelle réduite à celle d'un seul décideur, alors que nos résultats montrent son caractère distribué ; (ii) l'échelle de collecte et de traitement des informations considérée par les concepteurs est souvent la parcelle ${ }^{11}$, alors que l'activité décisionnelle met en jeu des échanges d'informations élaborées et/ou synthétisées à différentes échelles; (iii) le caractère séquentiel de l'activité décisionnelle est mal connu des concepteurs d'outils, qui souvent ne prennent en considération que les temporalités de la mesure.

Comment intégrer ces éléments dans la conception? Nous distinguons deux cas de figure : celui où la notion de SIC permet d'enrichir la réflexion des concepteurs sur les usages; celui où elle est mobilisée pour créer les

\footnotetext{
11 Le primat accordé à cette échelle est d'autant plus fort pour les concepteurs agronomes que la parcelle est l'objet fondateur, et toujours privilégié, de leur discipline (Sebillotte, 1974; Meynard et al., 2001).
} 
conditions d'une co-construction avec les utilisateurs des outils et des usages.

\section{Enrichir l'anticipation des utilisateurs, des usages et des utilisations au niveau de la conception}

Nous proposons ici de considérer les caractéristiques essentielles qui sous-tendent la notion de SIC pour concevoir des outils susceptibles de s'intégrer dans les formes concrètes que pourront avoir les SIC.

Quatre caractéristiques qualifiant un SIC nous apparaissent essentielles pour orienter ce renouvellement : la notion d'activité décisionnelle ; le caractère distribué de cette activité; les unités d'espace et de temps que les acteurs prennent en considération; les apprentissages que ces derniers développent.

La notion d'activité décisionnelle amène à considérer qu'un outil donné est en fait une source d'informations parmi d'autres pour appréhender une situation, concevoir une ou plusieurs solutions, sélectionner celle qui sera appliquée, l'évaluer. Les concepteurs gagneraient à s'appuyer d'emblée sur une analyse des outils disponibles pour positionner le leur. Si l'outil apparaît complémentaire de ceux qui existent, son mode de validation devra en tenir compte : un outil ne sera pas évalué de la même manière s'il sert à acquérir une information sur le statut azoté de la culture pour calibrer un modèle simulant les besoins en azote ultérieurs, ou s'il est utilisé pour décider d'une intervention en culture. De même, la validation d'un modèle épidémiologique diffère si celui-ci est utilisé pour déclencher un traitement phytosanitaire préventif sur l'ensemble des parcelles d'une région, ou s'il doit permettre d'identifier les parcelles sur lesquelles utiliser un kit pour ajuster le traitement à la parcelle. Lorsque l'outil ne présente pas de complémentarité avec ceux qui sont disponibles, il y a de fortes chances que son adoption s'accompagne de transformations importantes des modes de décision existants. Dans ce cas, l'accompagnement pour inscrire l'outil dans des formes locales de SIC demandera une attention particulière : les concepteurs devront s'interroger sur la façon d'aider les apprentissages de cet outil, soit en créant des supports, soit en déléguant le travail à des intermédiaires.

Le caractère distribué de l'activité décisionnelle, ainsi que les différences d'unités d'espace et de temps des acteurs impliqués dans un SIC, ont également des conséquences sur la validation des outils. Ainsi, pour un outil produisant une information à l'échelle de la parcelle, la validation sera différente dès lors que celle-ci participe à la production d'une information à validité régionale à partir d'un réseau de parcelles : à l'échelle parcellaire, il faudra s'assurer que l'information fournie par l'outil sépare bien deux populations de situations (celle où il faut agir et celle où il ne faut pas agir) ; à l'échelle régionale, il faudra s'assurer que l'information issue du réseau permet de discriminer correctement les différences interannuelles ou interrégionales d'évolution du phénomène cible; il faudra en outre valider la manière de constituer le réseau de parcelles. De plus, selon que l'outil aura pour vocation à être utilisé dans des situations atypiques ou, au contraire, dans tous les types de situations, le choix des cas de validation ne sera pas le même. Au-delà, c'est la façon même de construire, dans l'outil, le lien entre l'analyse des processus et l'action qui peut être questionnée. Comment tenir compte, par exemple, de la différence entre les objectifs d'un conseiller et ceux d'un agriculteur, ne serait-ce qu'au niveau strict de l'observation des processus? En effet, cette dernière est guidée par les finalités : le conseiller choisit des situations pour construire un conseil adapté à leur diversité au sein de la petite région; l'agriculteur, lui, cherche à évaluer la possibilité d'appliquer une "procédure de routine » ayant fait ses preuves (Cerf, 1996). Comment l'outil peut-il alors aider à construire ces procédures de routine et à reconnaître les situations où elles ne s'appliquent pas (Meynard et al., 2002)?

La reconnaissance de la créativité des utilisateurs a des conséquences sur les fonctionnalités à intégrer dans l'outil, en vue de lui conférer une plus grande souplesse d'utilisation. Une expérience intéressante est celle du logiciel PC-Azote, conçu initialement pour calculer des doses d'engrais, mais qui intègre la possibilité de mémoriser, dans un tableur, les calculs de fertilisation passés. Cette fonctionnalité a permis à l'outil de s'adapter à de nouveaux besoins : il est ainsi utilisé, à l'échelle de petits bassins de production, pour estimer des stocks de fertilisants à commander, faire un diagnostic sur les pratiques entraînant des risques de pollution nitrique ou assurer un suivi de l'évolution des pratiques (Angevin, 1999).

Ainsi, la connaissance des caractéristiques essentielles des SIC permet d'enrichir les débats entre concepteurs sur les fonctionnalités des nouveaux outils, les sorties attendues, les procédures de validation et les modes de représentation des interactions entre processus biophysiques et actions.

\section{Co-construire les usages et les outils}

Dans la perspective évoquée précédemment, l'activité constructive des acteurs est reléguée hors du processus de conception, n'étant qu'un élément susceptible de faciliter ou, au contraire, de réduire les usages des outils ainsi conçus. Peut-on imaginer une dynamique de conception qui l'intègre?

Une telle approche pourrait être particulièrement intéressante quand les outils envisagés constituent des évolutions notables pour les futurs utilisateurs. Le développement d'un outil destiné à éviter les traitements fongicides inutiles, par exemple contre le sclerotinia du colza, alors même qu'existent des traitements préventifs 
peu coûteux, peut s'avérer voué à l'échec si l'on ne tient pas compte du fait que les agriculteurs, comme leurs conseillers, considèrent qu'il est fortement risqué, dans leurs conditions habituelles de production, de ne pas traiter. Ainsi, tandis que concepteurs et développeurs des outils parlent de fréquence du risque (deux années sur dix) et visent l'absence de traitement dans les années où les épidémies sont limitées, les agriculteurs comme leurs conseillers parlent d'importance du risque (l'année où le sclerotinia n'est pas contrôlé, le rendement chute fortement) et visent un meilleur ajustement de la date de traitement (Cerf et Pasquier, 2004).

La mise en situation des futurs utilisateurs dans des scénarios où ils doivent faire des choix constitue un support à la construction de nouvelles ressources pour leur action, comme le montrent des travaux conduits dans d'autres secteurs (Béguin et Pastré, 2002; Amalberti, 1999). Dans le cas des travaux sur le sclerotinia, c'est à l'occasion de réunions autour de scénarios que les agriculteurs et leurs conseillers expriment la possibilité d'intégrer un outil de diagnostic, initialement proposé pour réduire la fréquence des traitements, dans un raisonnement de la date de traitement. De façon symétrique, les concepteurs ont, à l'issue de ces réunions, imaginé un système qui tente d'intégrer la façon dont les utilisateurs construisent le risque, tout en maintenant leur objectif de réduire au maximum les traitements inutiles. La mise en scénarios devient un support des apprentissages des uns et des autres et permet un développement conjoint des outils et de leurs usages.

Néanmoins, cette mise en situation se heurte à plusieurs difficultés. La première réside dans le choix des utilisateurs. Faut-il privilégier des acteurs susceptibles de devenir ensuite des relais auprès de leurs pairs, pour développer avec eux des apprentissages qu'ils pourront à leur tour susciter? Faut-il privilégier des acteurs représentant une cible particulière, comme les agriculteurs qui traitent systématiquement ou ceux qui sont localisés dans une zone écologiquement sensible? Faut-il, au contraire, rechercher la diversité des pratiques, tant au niveau des agriculteurs que des conseillers, afin d'organiser dans le processus de conception la confrontation d'expertises aussi diverses que possible? La deuxième difficulté réside dans le choix des situations qui serviront de support au dialogue entre utilisateurs et concepteurs. Comment définir des conditions agronomiques dans lesquelles se reconnaissent tous les acteurs impliqués dans la co-conception, alors qu'ils ont bâti leurs référentiels dans des situations différentes? Quel formalisme et quelles simplifications adopter? La troisième difficulté réside dans le choix des contextes de l'interaction concepteur/utilisateur. Faut-il travailler avec un utilisateur isolé, ou bien faut-il faire fonctionner un groupe potentiel, susceptible de s'organiser localement pour l'utilisation des nouveaux outils? Faut-il s'appuyer sur un SIC existant?
Faut-il s'inscrire à un moment-clé dans le processus de conduite pour faciliter la mise en situation?

Derrière ces questions, ce n'est pas uniquement la prise en considération de l'activité constructive des acteurs qui est en jeu. C'est, d'une part, la façon dont les agronomes peuvent contribuer à mettre en forme les connaissances sur les processus pour créer les conditions de mises en situation susceptibles de faciliter l'implication de différentes expertises (du chercheur, du conseiller, de l'agriculteur, par exemple) dans un processus de conception. C'est, d'autre part, la façon dont on se représente les processus d'innovation et de changement technique. Dans le monde agricole, la variabilité de situations de travail est prodigieuse et seul le monde médical en offre sans doute autant. Dès lors, la conception d'outils s'apparente davantage au travail des concepteurs de produits grand public qu'à celui d'un bureau des méthodes dans une entreprise. À cela s'ajoute la présence d'un "corps intermédiaire », celui des prescripteurs, qui non seulement diffuse de nouvelles technologies vers les agriculteurs, mais les utilise pour construire son conseil ou pour développer des systèmes d'information et de conseil orientés vers l'aide à la décision des agriculteurs. La façon dont s'est organisé le «développement agricole» depuis les années 1960 marque profondément les processus d'innovation en agriculture. Or, dans la conception, les conseillers ont un rôle de médiation insuffisamment reconnu. Une plus grande compréhension de leur travail devrait permettre d'avancer dans une meilleure appréhension des SIC et de leurs effets sur la dynamique des outils.

\section{Références}

Akrich, M., 1993. Les objets techniques et leurs utilisateurs, Raisons pratiques, 4, 35-57.

Amalberti, R., 1999. Les Systèmes à risques, Paris, PUF.

Angevin, F., 1999. Raisonnement de la fertilisation azotée : le logiciel PC-Azote en Poitou-Charentes, Oléoscope, 54, 32-34.

Béguin, P., 2003. Design as a mutual learning process between users and designers, Interacting with Computers, 15, 5, 709730.

Béguin, P., Rabardel, P., 2000. Designing for instrument mediated activity, Scandinavian Journal of Information Systems, 12, 173-190.

Béguin, P., Pastré, P., 2002. Working, learning and designing through simulation, in Bagnara, S., Pozzi, S., Rizzo, A., Wright, P. (Eds), Proceedings of the 11th European Conference on Cognitive Ergonomics: Cognition, Culture and Design, Catania, Italy, September 8-11, 5-13.

Bødker, S., Grønboek, K., 1996. Users and designers in mutual activity: an analysis of cooperative activities in systems design, in Engeström, Y., Middleton, D. (Eds), Cognition and Communication at Work, Cambridge, Cambridge University Press, 87-102. 
Bratteteig, T., 1997. Mutual learning. Enabling cooperation on systems design, in Bras, K., Monteiro, E. (Eds), Procedings of IRIS 20, University of Oslo, June 1997, 1-19.

Cerf, M., 1996. Approche cognitive des pratiques agricoles : intérêts et limites pour les agronomes, Natures Sciences Sociétés, 4, 4, 327-339.

Cerf, M., Meynard, J.-M., 1988. Enquête sur la mise en œuvre des méthodes de fertilisation raisonnée, $3^{e}$ forum national de la fertilisation raisonnée, Nancy, COMIFER, 5-12.

Cerf, M., Pasquier, C., 2004. Intégrer les usages dans la conception d'outils d'aide au raisonnement des traitements contre sclerotinia. Rapport de fin de recherches, Thiverval-Grignon, INRA, UMR SADAPT.

Cultivar, 2002. Présence de maladies fongiques sur blé : évaluer le risque à la parcelle, Cultivar-Grandes cultures, 543, 20-21.

Emonet, E., 1998. La Communication entre agriculteurs et prescripteurs à travers l'utilisation des outils de pilotage du blé et du colza. Mémoire de DAA, sous la direction de M. Cerf et J.-M. Meynard, ENSAR, Rennes.

Felix, I., Reau, R., 1995. Pratiques de fertilisation : un constat paradoxal!, Perspectives agricoles, 207, 65-69.

Fixari, D., Spira, R. 1998. La méthode JUBIL pour la maîtrise de la fertilisation azotée, in Aggeri, F., et al. (Eds), Les Chercheurs et l'innovation: regards sur les pratiques de l'INRA, Paris, INRA Éditions, 325-343.

Hébinger, H., 1994. Simple comme une cuvette jaune, Magasin agricole, janvier, 38-39.

Hoc, J.-M., 1989. La Planification, Grenoble, PUG.

Hutchins, E., 1995. Cognition in the Wild, Cambridge, The MIT Press.

Hydro-Agri, 1997. Diagnostic Hydro N-Tester; Guide technique Fertilisation blé.

ITCF, 1978. La Fertilisation azotée du blé. Brochure, Paris.

Jeuffroy, M-H., Meynard, J-M., Sevat, B., 1987. Identification des obstacles à la mise en œuvre des méthodes de fertilisation raisonnée : une enquête réalisée par le COMIFER avec les lycées agricoles, Bulletin technique d'information, 420, 241-245.

Justes, E., 1993. Diagnostic de la nutrition azotée du blé à partir de la teneur en nitrate du jus de la base de la tige : application au raisonnement de la fertilisation. Thèse de doctorat, INA P-G, Paris.

Justes, E., Meynard, J-M., Mary, B., Plenet, D., 1997. Diagnosis using stem base extract: JUBIL Method, in Lemaire, G. (Ed.), Diagnosis of the Nitrogen Status in Crops, Berlin, SpringerVerlag, 163-187.

Kyng, M., 1995. Creatring contexts for design, in Carroll, J.M. (Ed.), Scenario-Based Design: Envisionning Work and Technology in System Development, New York, John Wiley \& Sons, 85-108.

Laurent, F., Justes, E., Gate, P., 1996. JUBIL 1996 : la méthode s'affine, Perspectives agricoles, 214, 63-74.

Limaux, F., Aubry, C., Bourzeix, A., Constant, J.-B., 1993. Fertilisation azotée du blé en Lorraine : constats et proposition de nouvelles pratiques, Perspectives agricoles, 179, 58-73.

Limaux, F., Meynard, J.-M., Recous, S., 2001. Le témoin «doubledensité » pour déclencher la fertilisation azotée du blé : bases théoriques et principes généraux, Perspectives agricoles, 273, 62-70.
Loyce, C., Rellier, J-P., Meynard, J-M., 2001. Management planning for winter wheat with multiple objectives (1): The BETHA system, Agricultural Systems, 72, 9-31.

Machet, J.-M., Dubrulle, P., Louis, P., 1990. AZOBIL: a computer program for fertilizer $\mathrm{N}$ recommendations based on a predictive balance sheet method, Proceedings of the 1st Congress of the European Society of Agronomy.

Meynard, J-M., Justes, E., Machet, J-M., Recous, S., 1997. Fertilisation azotée des cultures annuelles de plein champ, in Lemaire, G, Nicolardot, B. (Eds), Maîtrise de l'azote dans les agrosystèmes, Paris, INRA Éditions, 271-288.

Meynard, J-M., Doré, T., Habib, R., 2001. L'évaluation et la conception de systèmes de culture pour une agriculture durable, C.R. Acad. Agric. Fr., 87, 4, 223-236.

Meynard, J-M., Cerf, M., Guichard, L., Jeuffroy, M-H., Makowski, D., 2002. Which decision support tools for the environmental management of nitrogen?, Agronomie, 22, 7/8, 817-829.

Murer, F., Rouzet, J., 1997. Lutte raisonnée : les différents apports des modèles de prévision selon les parasites et les cultures, Cinquième conférence internationale sur les maladies des plantes, ANPP, 47-54.

Orlikowski, W.J., Robey, D., 1991. Information technology and the structuring of organizations, Information Systems Research, 2, 143-169.

Plas, D., 1992. Adaptation régionale du raisonnement de la fertilisation azotée : cas du blé tendre en Aunis. Thèse de doctorat, Université technologique de Compiègne.

Plas, D., Meynard, J-M., Delphin, A., Taureau, J-C., Bouthier, A., 1994. Terres de Groie : un raisonnement original de la fertilisation azotée, Perspectives agricoles, 97, 73-77.

Rabardel, P., 1995. Les Hommes et les technologies : approche cognitive des instruments contemporains, Paris, Armand Colin.

Reau, R., Jung, L., Wagner, D., 1997. Les bases de la fertilisation azotée prévisionnelle, Oléoscope, 20, 29-37.

Reibel, C., 1996. Des outils traquent la septoriose, Cultivar, 420, 26-27.

Rémy, J-C., Hébert, J., 1977. Le devenir des engrais dans le sol, C.R. Acad. Agric. Fr., 63, 700-710.

Samurçay, R., Rabardel, P., 1995. Work competencies: some reflections for a constructivist theoretical framework, in Proceedings 2nd Work Process Knowledge Meeting: Theoretical Approaches of Competences at Work, Courcelle-sur-Yvette, France, October 19-21.

Sebillotte, M., 1974. Agronomie et Agriculture. Essai d'analyse des tâches de l'agronome, Cahiers ORSTOM, série Biologie, 24, 3-25.

Sebillotte, M., Soler, L.G., 1988. Le concept de modèle général et la compréhension du comportement de l'agriculteur, C.R. Acad. Agric. Fr., 74, 59-70.

Soulard, C., 2005. Les agriculteurs et la pollution des eaux. Proposition d'une géographie des pratiques, Natures Sciences Sociétés,13, 2, 154-164.

Suchman, L., 1987. Plans and Situated Actions: The Problem of Human Machine Interaction, Cambridge, Cambridge University Press.

Taverne, M., 2000. Contribution à la conception d'un système d'aide à la décision pour les traitements contre le sclerotinia du colza. Mémoire de DESS, sous la direction de M. Cerf et J.M. Meynard, Université de Clermont-Ferrand - ENITAC, Clermont-Ferrand. 
Annexe. Présentation des outils étudiés

\begin{tabular}{|c|c|c|c|c|c|}
\hline $\begin{array}{l}\text { Nom de } \\
\text { l'outil }\end{array}$ & $\begin{array}{l}\text { Composante } \\
\text { matérielle }\end{array}$ & $\begin{array}{l}\text { Choix } \\
\text { technique } \\
\text { concerné }\end{array}$ & $\begin{array}{l}\text { Procédure } \\
\text { d'utilisation }\end{array}$ & Inventeur & Référence \\
\hline AZOBIL & $\begin{array}{l}\text { Logiciel de calcul } \\
\text { des doses d'engrais, } \\
\text { basé sur un bilan } \\
\text { prévisionnel d'azote }\end{array}$ & $\begin{array}{l}\text { Fertilisation } \\
\text { azotée } \\
\text { cultures } \\
\text { annuelles }\end{array}$ & $\begin{array}{l}\text { Saisie d'informations } \\
\text { sur la parcelle dont } \\
\text { une mesure d'azote } \\
\text { minéral du sol en fin } \\
\text { d'hiver }\end{array}$ & $\begin{array}{l}\text { Inra et Station } \\
\text { agronomique de } \\
\text { l'Aisne }\end{array}$ & $\begin{array}{l}\text { Machet } \text { et al., } \\
1990\end{array}$ \\
\hline PC Azote & $\begin{array}{l}\text { Logiciel de calcul des } \\
\text { doses d'engrais }\end{array}$ & $\begin{array}{l}\text { Fertilisation } \\
\text { azotée } \\
\text { cultures } \\
\text { annuelles }\end{array}$ & $\begin{array}{l}\text { Saisie d'informations } \\
\text { parcellaires (type de } \\
\text { sol, histoire de la } \\
\text { parcelle) et locales } \\
\text { (pluviométrie) }\end{array}$ & $\begin{array}{l}\text { Agrotransfert } \\
\text { Poitou-Charentes }\end{array}$ & $\begin{array}{l}\text { Plas, 1992; } \\
\text { Plas et al., } \\
1994 \text {; } \\
\text { Angevin, } \\
1999\end{array}$ \\
\hline JUBIL & $\begin{array}{l}\text { Mallette JUBIL, } \\
\text { contenant en } \\
\text { particulier un } \\
\text { analyseur de nitrate } \\
\text { dans la plante }\end{array}$ & $\begin{array}{l}\text { Fertilisation } \\
\text { azotée du } \\
\text { blé }\end{array}$ & $\begin{array}{l}\text { Calcul de dose } \mathrm{N} \text { par } \\
\text { bilan prévisionnel; } \\
\text { puis suivi de la teneur } \\
\text { en nitrate des plantes } \\
\text { pour appliquer ou non } \\
\text { un complément }\end{array}$ & $\begin{array}{l}\text { Inra et ITCF pour la } \\
\text { méthode de } \\
\text { fertilisation; } \\
\text { Challenge } \\
\text { Agriculture pour la } \\
\text { mallette }\end{array}$ & $\begin{array}{l}\text { Justes et al., } \\
1997 ; \\
\text { Laurent } \\
\text { et al., } 1996\end{array}$ \\
\hline $\begin{array}{l}\text { Hydro N- } \\
\text { Tester }\end{array}$ & $\begin{array}{l}\text { Pince combinée à } \\
\text { cellule } \\
\text { photométrique pour } \\
\text { mesurer la teneur } \\
\text { en chlorophylle des } \\
\text { feuilles et logiciel } \\
\text { d'interprétation }\end{array}$ & $\begin{array}{l}\text { Fertilisation } \\
\text { azotée du } \\
\text { blé }\end{array}$ & $\begin{array}{l}\text { Calcul de dose N par } \\
\text { bilan prévisionnel, } \\
\text { puis suivi de la } \\
\text { teneur en chlorophylle } \\
\text { des feuilles pour } \\
\text { appliquer ou non un } \\
\text { complément }\end{array}$ & Hydro-Agri & $\begin{array}{l}\text { Hydro-Agri, } \\
1997\end{array}$ \\
\hline $\begin{array}{l}\text { Réglette } \\
\text { Azote } \\
\text { Colza }\end{array}$ & $\begin{array}{l}\text { Réglette avec curseur } \\
\text { et abaques } \\
\text { permettant le calcul } \\
\text { d'un bilan } \\
\text { prévisionnel }\end{array}$ & $\begin{array}{l}\text { Fertilisation } \\
\text { azotée du } \\
\text { colza }\end{array}$ & $\begin{array}{l}\text { Saisie d'informations } \\
\text { parcellaires, dont } \\
\text { pesée du colza en fin } \\
\text { d'hiver }\end{array}$ & CETIOM & $\begin{array}{l}\text { Reau et al., } \\
1997\end{array}$ \\
\hline POSITIF & $\begin{array}{l}\text { Logiciel d'estimation } \\
\text { du risque local de } \\
\text { maladies fongiques }\end{array}$ & $\begin{array}{l}\text { Fongicides } \\
\text { blé }\end{array}$ & $\begin{array}{l}\text { Saisie du climat local } \\
\text { au jour le jour }\end{array}$ & Rhône-Poulenc & $\begin{array}{l}\text { Reibel, C, } \\
1996\end{array}$ \\
\hline CLEAN & $\begin{array}{l}\text { Logiciel d'estimation } \\
\text { du risque local de } \\
\text { maladies fongiques }\end{array}$ & $\begin{array}{l}\text { Fongicides } \\
\text { blé }\end{array}$ & $\begin{array}{l}\text { Saisie du climat local } \\
\text { au jour le jour et des } \\
\text { stades de la culture }\end{array}$ & $\begin{array}{l}\text { Service de la } \\
\text { protection des } \\
\text { végétaux }\end{array}$ & $\begin{array}{l}\text { Murer et } \\
\text { Rouzet, } 1997\end{array}$ \\
\hline $\begin{array}{l}\text { Kit } \\
\text { Diagnolab }\end{array}$ & $\begin{array}{l}\text { Kit de détection du } \\
\text { piétin-verse sur tige, } \\
\text { basé sur } \\
\text { immunologie et règle } \\
\text { d'interprétation }\end{array}$ & $\begin{array}{l}\text { Fongicide } \\
\text { contre le } \\
\text { piétin-verse } \\
\text { du blé }\end{array}$ & $\begin{array}{l}\text { Prélèvement de } \\
\text { plantes au niveau de } \\
\text { chaque parcelle à un } \\
\text { stade précis, analyse } \\
\text { centralisée }\end{array}$ & Dupont de Nemours & \\
\hline $\begin{array}{l}\text { Grille de } \\
\text { risque } \\
\text { Piétin- } \\
\text { verse }\end{array}$ & $\begin{array}{l}\text { Tableau de calcul } \\
\text { d'une note de risque } \\
\text { de piétin-verse } \\
\text { et règles } \\
\text { d'interprétation }\end{array}$ & $\begin{array}{l}\text { Fongicide } \\
\text { contre le } \\
\text { piétin-verse } \\
\text { du blé }\end{array}$ & $\begin{array}{l}\text { Calcul alimenté par la } \\
\text { connaissance du type } \\
\text { de sol, de l'histoire de } \\
\text { la parcelle, de la date } \\
\text { de semis et de la } \\
\text { sensibilité de la variété }\end{array}$ & $\begin{array}{l}\text { ITCF (devenu } \\
\text { ARVALIS-Institut du } \\
\text { végétal) }\end{array}$ & $\begin{array}{l}\text { ITCF Centre- } \\
\text { Île-de-France, } \\
\text { cité par } \\
\text { Cultivar, } \\
2002\end{array}$ \\
\hline $\begin{array}{l}\text { Cuvette } \\
\text { jaune }\end{array}$ & $\begin{array}{l}\text { Piège à insectes et } \\
\text { règles } \\
\text { d'interprétation du } \\
\text { comptage d'insectes } \\
\text { piégés }\end{array}$ & $\begin{array}{l}\text { Insecticides } \\
\text { Colza }\end{array}$ & $\begin{array}{l}\text { Placement du piège } \\
\text { dans une parcelle, } \\
\text { surveillance } \\
\text { hebdomadaire et } \\
\text { comptage des } \\
\text { insectes; intervention } \\
\text { sur seuil }\end{array}$ & CETIOM & $\begin{array}{l}\text { Hébinger, } \\
1994\end{array}$ \\
\hline
\end{tabular}

